\title{
Prevalence of Neoplasms in patients with Primary Aldosteronism
}

K. Lang, K. Weber, M. Quinkler, A. Pallauf, H. Wallaschofski, A. Hannemann, I. Quack, L.-C.Rump, H. Willenberg,

M. Reincke, B. Allolio, S. Hahner

Context: Primary aldosteronism (PA) is the most common cause of secondary hypertension. In vitro, aldosterone excess can cause oxidative stress leading to DNA damage. Single case reports describe a coincidence of PA with renal cell carcinoma and other tumors. However, no data on the prevalence of benign and malignant neoplasms in patients with PA exists.

Methods: In the multicentre MEPHISTO study the prevalence of benign and malignant tumors was investigated in 338 patients both pro- and retrospectively. The SHIP cohort of patients with essential hypertension served as a matched control group.

\section{Neoplasms in PA patients}

\begin{tabular}{|l|r|}
\hline benign & 98 (61,6\%) \\
\hline endocrine tissue & $\mathbf{3 7}(\mathbf{3 8 \%})$ \\
prostate & $\mathbf{2 1 ( 2 1 \% )}$ \\
skin & $\mathbf{6}(\mathbf{6} \%)$ \\
gynaecologic & $\mathbf{1 1 ( 1 1 \% )}$ \\
others & $\mathbf{2 3 ( 2 3 \% )}$ \\
\hline malignant & $39(24,5 \%)$ \\
\hline urinary tract & $\mathbf{1 1 ( 2 8 \% )}$ \\
renal cell carcinoma & $5(13 \%)$ \\
prostate cancer & $6(15 \%)$ \\
skin & $\mathbf{8 ( 2 1 \% )}$ \\
gynaecologic & $\mathbf{6 ( 1 5 \% )}$ \\
others & $\mathbf{1 4}(36 \%)$ \\
\hline unknown dignity & $22(13,8 \%)$ \\
\hline
\end{tabular}

- 338 patients with confirmed primary aldosteronism (PA)

$$
n=199(59 \%) \quad n=139(41 \%)
$$

$\rightarrow$ total neoplasms $\mathbf{n}=159$

\section{Benign endocrine neoplasms in PA patients}

Aldosterone tertiles

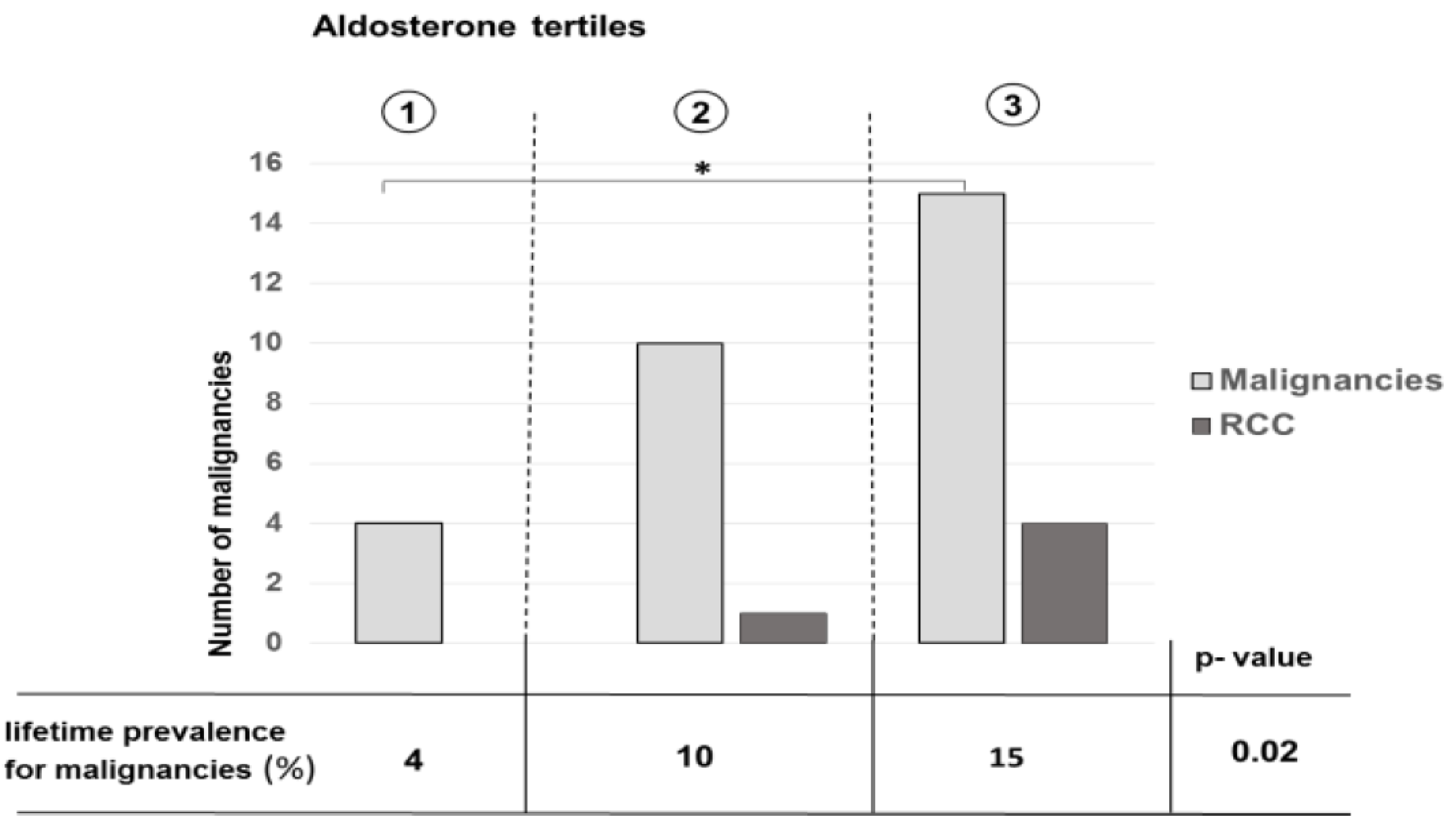

$\rightarrow$ positive correlation between Aldosterone levels and lifetime prevalance of malignancies

\section{SHIP-1 as a matched control group}

- 335 MEPHISTO patients

3 patients $<27$ yr excluded)

- Hypertensive control group $\rightarrow 1$ :1 matching for age and sex

\begin{tabular}{|l|l|l|l|}
\hline & MEPHISTO & SHIP-1 & P \\
\hline Patients with cancer & 32 & 20 & $\mathbf{0 . 0 8}$ \\
\hline Renal cell carcinoma & 5 & 0 & \\
\hline Thyroid cancer & 3 & 1 & \\
\hline BMI (kg/m $\left.{ }^{2}\right)$ & 28.1 & 28.5 & $\mathbf{0 . 2 8}$ \\
\hline active smokers $(\mathbf{n})$ & 50 & 72 & \\
\hline
\end{tabular}

\section{Comparison of Aldosterone levels at diagnosis of PA}

\begin{tabular}{|c|c|c|c|}
\hline $\begin{array}{l}\text { Aldosterone } \\
\text { (ng/l) }\end{array}$ & $\begin{array}{l}\text { Individuals with } \\
\text { malignancies }\end{array}$ & $\begin{array}{l}\text { Individuals without } \\
\text { malignancies }\end{array}$ & $\begin{array}{l}p- \\
\text { value }\end{array}$ \\
\hline $\begin{array}{l}\text { Primary } \\
\text { aldosteronism }\end{array}$ & $350(244.5-509.5)$ & $239.2(151.3-379.6)$ & 0.009 \\
\hline $\begin{array}{l}\text { Controls (SHIP- } \\
\text { 1) }\end{array}$ & $56.5(32.5-71)$ & $42.0(28.0-65.0)$ & 0.17 \\
\hline
\end{tabular}

\section{Renal cell carcinoma ( $R C C$ ) in PA patients}

$\rightarrow$ RKI cancer registry (2006): $3.3-4.4 \%$ of all malignancies $\rightarrow$ MEPHISTO: $\quad 13 \%$ of all malignancies

Literature research:

- 16 - $44 \%$ presence of renal cysts in PA patients

- aquired renal cysts are independently associated with RCC

$\rightarrow$ MEPHISTO: 5 RCC

$\cdot 80 \%$

$\cdot 80 \%$ unilateral disease

-all hypokalemic at diagnosis of PA

-1 tumor incidentally discovered

$.80 \%$ with history of renal cysts

$\rightarrow$ highest aldosterone levels !

Discussion: In this cohort of PA patients a trend towards an increased lifetime prevalence for malignancies was observed. Formation of malignancies correlated significantly with baseline aldosterone levels, which was particularly obvious for renal cell carcinomas. Increased prevalence for renal cell carcinomas with renal cysts as possible predisposition will have to be confirmed and further investigated. 